Introduction to the Special Issue

\title{
Service Innovations for E-commerce
}

\author{
Christopher C. Yang \\ College of Information Science and \\ Technology \\ Drexel University \\ Philadelphia, Pennsylvania, USA \\ chris.yang@drexel.edu
}

\author{
Chih-Ping Wei \\ Department of Information Management \\ College of Management \\ National Taiwan University \\ Taipei, Taiwan, ROC \\ cpwei@im.ntu.edu.tw
}

\section{Introduction}

With rapid advancements of information and communication technologies and its integration with business, e-commerce has become common practices for many business transactions. It has emerged with business processes to deliver values to internal and external customers. The demand of customer-centric services requires innovative approaches to identify customer needs, and design effective processes to deliver values to customers, especially facing the increasing number of Web-based applications (e.g., Web 2.0). Thus, the study of the innovation in services for e-commerce is becoming the next wave of e-commerce research and practices. This special issue aims to advance our understanding of this matter and hopefully to encourage and develop research momentum across disciplines to contribute to service innovations for e-commerce.

In this special issue, four papers are selected to cover the design of customer expectation measurement model and a range of e-commerce service innovations. These papers were chosen, extended, and further revised from those presented at the Eleventh International Conference on Electronic Commerce (ICEC 2009) in August 2009 in Taipei, Taiwan.

The special issue begins with Hsieh and Yuan's paper, "Design of the Customer Expectation Measurement Model in Dynamic Service Experience Delivery." The authors propose a systematical and quantitative expectation measurement model for customer-focused service design and innovation. The proposed model is developed on the basis of Fechner's Law and concepts of operation risk. Such model provides a real time measurement which overcomes the limitation of the traditional empirical approach.

In the second paper, "A Ubiquitous Knowledge-based System to Enable RFID Object Discovery in Smart Environments," Ruta, Di Sciascio, Piscitelli, and Scioscia develop an extended framework to enable the ubiquitous Knowledge Base (u-KB) system in mobile environments. In their framework, semantic matchmaking is carried out using metadata in Radio Frequency Identification (RFID) tags. An evaluation using ns-2 simulation environment is conducted to test the feasibility of the proposed framework.

A large volume of online reviews are available but the quality varies. In response, Huang, Shen, Feng, Baudin, and Zhang, in their paper on "Promote Product Reviews of High Quality on Ecommerce Sites," explore two aspects, including noise filtering and author behavior, to discover the product reviews of high quality. In noise filtering, reviews are classified into product reviews, feedbacks to retailers, and commercial spams. Three behavior features pertaining to online reviewers, including personal reputation, seller degree, and expertise degree, are investigated for ranking the product reviews. A behavior model is developed to capture user behavior in making product reviews and voting on others' reviews. 
The special issue ends with a paper that investigates sentiment analysis of online consumer reviews in a Web 2.0 environment. In "Understanding Online Consumer Review Opinions with Sentiment Analysis using Machine Learning," Yang, Tang, Wong, and Wei employ machine learning techniques, namely class association rules and naïve Bayes classifier, to classify online consumer review opinions into product feature classes and generate a summary of consumer reviews. Such techniques help users to identify products with good reviews on the preferred product features effectively and efficiently rather than relying on the general ratings. The investigated techniques overcome the shortcomings of the natural language processing approach. The empirical evaluation shows that their proposed techniques achieve over $70 \%$ of macro and micro F-measures.

\section{Acknowledgement}

The guest editors wish to thank Professor Ting-Peng Liang, Editor-in-Chief of the journal, who has provided strong support for this special issue. To all who involved in organizing ICEC 2009, we deeply appreciate their great contributions to making a successful conference that provides a great foundation for this special issue. Our special thanks go to all reviewers of this special issue for their valuable comments and suggestions. We also appreciate the contributing authors' willingness to permit us to shape their works during the review process for those papers submitted to ICEC 2009 and the regular review process of the journal.

\section{About the Guest Editors}

Christopher C. Yang is an associate professor in the College of Information Science and Technology at Drexel University. He has also been an associate professor in the Department of Systems Engineering and Engineering Management and the director of the Digital Library Laboratory at the Chinese University of Hong Kong, an assistant professor in the Department of Computer Science and Information Systems at the University of Hong Kong and a research scientist in the Department of Management Information Systems at the University of Arizona. His recent research interests include social media analytics, Web 2.0, security informatics, health informatics, Web search and mining, knowledge management, and electronic commerce. $\mathrm{He}$ has published over 200 referred journal and conference papers in Journal of the American Society for Information Science and Technology (JASIST), Decision Support Systems (DSS), IEEE Transactions on Systems, Man, and Cybernetics, IEEE Transactions on Image Processing, IEEE Transactions on Robotics and Automation, IEEE Computer, IEEE Intelligent Systems, Information Processing and Management (IPM), Journal of Information Science, Graphical Models and Image Processing, Optical Engineering, Pattern Recognition, International Journal of Electronic Commerce, Applied Artificial Intelligence, ISI, WWW, SIGIR, ICIS, CIKM, and more. He has edited several special issues on multilingual information systems, knowledge management, Web mining, social media, and electronic commerce in JASIST, DSS, IPM, and IEEE Transactions. He chaired and served in many international conferences and workshops. $\mathrm{He}$ has also frequently served as an invited panelist in the NSF and other government agencies review panels. He can be reached at chris.yang@drexel.edu.

Chih-Ping Wei received a BS in Management Science from the National Chiao-Tung University in Taiwan, R.O.C. in 1987 and an MS and a Ph.D. in Management Information Systems from the University of Arizona in 1991 and 1996. He is currently a professor of Department of Information Management at National Taiwan University. Prior to joining National Taiwan University in 2010, he was a professor of Institute of Service Science and Institute of Technology Management at National Tsing Hua University in Taiwan and a professor of Department of Information Management at National Sun Yat-sen University in Taiwan. He was also a visiting scholar at the University of Illinois at Urbana-Champaign in Fall 2001 and the Chinese University of Hong Kong in Summer 2006 and 2007. His papers have appeared in Journal of Management Informa- 
tion Systems (JMIS), European Journal of Information Systems, Decision Support Systems (DSS), IEEE Transactions on Engineering Management, IEEE Software, IEEE Intelligent Systems, IEEE Transactions on Systems, Man, Cybernetics, IEEE Transactions on Information Technology in Biomedicine, Journal of the American Society for Information Science and Technology, Information Processing and Management, Journal of Database Management, and Journal of Organizational Computing and Electronic Commerce, etc. His current research interests include information retrieval and text mining, knowledge discovery and data mining, knowledge management, multidatabase management and integration, and data warehouse design. He has edited special issues of Decision Support Systems, International Journal of Electronic Commerce, Electronic Commerce Research and Applications, and Information Processing and Management. He can be reached at the Department of Information Management, National Taiwan University, Taipei, Taiwan, R.O.C; cpwei@im.ntu.edu.tw. 